\title{
Motion-based prediction of external forces and moments and back loading during manual material handling tasks
}

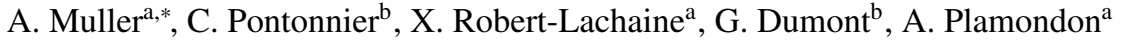 \\ ${ }^{a}$ Institut de Recherche Robert-Sauvé en Santé et en Sécurité du Travail (IRSST), Montréal, QC, Canada \\ ${ }^{b}$ Univ Rennes, CNRS, Inria, IRISA - UMR 6074, M2S, 35042 Rennes, France
}

\begin{abstract}
This paper evaluates a method for motion-based prediction of external forces and moments on manual material handling (MMH) tasks. From a set of hypothesized contact points between the subject and the environment (ground and load), external forces were calculated as the minimal forces at each contact point while ensuring the dynamics equilibrium. Ground reaction forces and moments (GRF\&M) and load contact forces and moments (LCF\&M) were computed from motion data alone. With an inverse dynamics method, the predicted data were then used to compute kinetic variables such as back loading. On a cohort of 65 subjects performing MMH tasks, the mean correlation coefficients between predicted and experimentally measured GRF for the vertical, antero-posterior and medio-lateral components were $0.91(0.08), 0.95(0.03)$ and $0.94(0.08)$, respectively. The associated RMSE were $0.51 \mathrm{~N} / \mathrm{kg}, 0.22 \mathrm{~N} / \mathrm{kg}$ and $0.19 \mathrm{~N} / \mathrm{kg}$. The correlation coefficient between L5/S1 joint moments computed from predicted and measured data was 0.95 with a RMSE of $14 \mathrm{Nm}$ for the flexion / extension component. In conclusion, this method allows the assessment of MMH tasks without force platforms, which increases the ecological aspect of the tasks studied and enables performance of dynamic analyses in real settings outside the laboratory.
\end{abstract}

Keywords: Ground reaction forces and moments, lifting, kinematics, inverse dynamics, L5/S1 joint moment

\section{Introduction}

The National Research Council (2001) reported that there is a clear relationship between back disorders and physical load. In this context, numerous studies have evaluated back loading in manual materials handling (MMH) (Marras et al. 2006; Plamondon et al., 2010; Ning et al. 2014, Corbeil et al., 2019, Gagnon et al. 2018). In most of these studies, back loading was assessed from the L5/S1 joint moments, based on a motion capture system associated to one or more force platforms, usually in a motion analysis laboratory. The use of such devices reduces the ecological aspect of the tasks (i.e. close to workplace conditions) by constraining the analysis area to the force platforms. To remove this barrier, several studies have been proposed that predict the external forces and moments from motion data only. In the great majority of studies, the external forces and moments were limited to the ground reaction forces and moments (GRF\&M). They could be estimated by directly solving the inverse dynamics problem (Delisle et al., 1999, Faber et al., 2016). However, this method uses only one global contact since the problem is undetermined with multiple contacts. Three

\footnotetext{
${ }^{*}$ Corresponding author

Email address: antoine.muller@irsst.qc.ca (A. Muller)
}

motion-based prediction approaches to solve this indeterminacy have been identified in the literature.

A first approach uses empirical functions to link the motion and associated GRF\&M (Koopman et al., 1995, Dijkstra and Gutierrez-Farewik, 2015). This approach has only been applied on gait.

A second approach is based on machine learning methods (Choi et al., 2013; Oh et al., 2013, Johnson et al., 2018). From data set using force platforms, a neural network establishes a link between the motion and the GRF\&M. These methods, based on a learning phase, have the disadvantage of requiring a set of motions close to the one studied and thus are only applicable to standardized motions. In addition, a large motion set is needed for the learning phase to be efficient.

A third approach uses a contact model with optimization techniques (Fluit et al., 2014, Jung et al. 2016; Skals et al., 2017), where a set of discrete contact points is defined on a biomechanical model (corresponding to the hypothesized contact points between the subject and the environment). Then, the external forces are calculated as the minimal forces for each contact point while ensuring respect for the dynamics equations. This approach was recently evaluated on MMH tasks (Larsen and Svenningsen, 2018; Muller et al. 2019a) and the results were promising. However, these studies were limited to standardized tasks: loads 
were carried only using handles and tasks did not require moving the feet. The objective of this study was to evaluate a motion-based prediction method based on a contact model on more ecological MMH tasks. The experimental procedures are detailed below. Based only on motion data, the prediction method estimates the GRF\&M and the load contact forces and moments (LCF\&M). More specifically, the method evaluation consists in first, comparing the predicted GRF\&M to force platform data and, second, comparing predicted back loading with the L5/S1 joint moments to those computed by using the force platform data.

\section{Materials and methods}

\subsection{Experimental procedure}

Several studies analyzing MMH tasks have been carried out at the IRSST (Institut de recherche RobertSauvé en santé et en sécurité du travail, Montréal, Canada) biomechanical laboratory. They consisted in comparing expert and novice workers (Plamondon et al. 2010), analyzing the importance of expertise, lifting height and weight lifted (Plamondon et al., 2012), comparing healthy bodyweight and obese workers (Corbeil et al. 2013, 2019) and comparing male and female workers (Gagnon et al., 2018). The parameters studied were kinematic variables such as the upper trunk flexion, horizontal hand distance to L5/S1 or knee flexion and kinetic variables such as the L5/S1 joint moments. The experimental protocol was the same for each and is detailed below.

The cohort was composed of 65 subjects $(50$ males, 15 females, age: $27 \pm 7$ years old, height: $177 \pm 4 \mathrm{~cm}$, mass: $73 \pm 15 \mathrm{~kg}$ ). The variability of the subjects (men / women, healthy bodyweight / obese, experts / novices) allows to take into account the variability of the handlers in companies. The studies were approved by the local institution's research ethics committee. Each participant signed an informed consent form prior to participation. An Optotrak system (Northern Digital Inc., Waterloo, Ontario, Canada) recorded at $30 \mathrm{~Hz}$ the 3-D coordinates of markers located on the subject. As previously done (Plamondon et al., 2010, 2012; Corbeil et al., 2013, 2019, Gagnon et al., 2018), twelve clusters of four markers were attached to each subject segment (head, back at C7, T12 and S1, as well as both arms, both forearms, both thighs and both feet). At the beginning of each experiment, the locations of 48 anatomical landmarks were identified in relation to their respective cluster. The GRF were measured by an extended force platform $(1.90 \times 1.40 \mathrm{~m})$ mounted on six AMTI mini platforms (model MC3A-6-1000; Advanced Mechanical Technology Inc., Watertown, MA, USA). The force platform collected data at $1024 \mathrm{~Hz}$ and had been previously validated (Desjardins and Gagnon, 2001).
The task consisted in transferring four boxes one by one from a conveyor (height from the ground $12 \mathrm{~cm}$ ) to a two-wheel hand trolley (height from the ground $2 \mathrm{~cm}$ ) and back again (Figure 11). The distance between the lifting and deposit locations was $1.5 \mathrm{~m}$. In the initial phase to the hand trolley, the conveyor was slightly inclined so that the boxes moved towards the handler. The four boxes were transferred one by one to be stacked in a pile on the hand trolley. In the return phase to the conveyor, the conveyor was slightly inclined outwards so that the boxes moved away from the handler. No constraint on foot motion was imposed on the subject. 16 repetitions of the initial and of the return phase resulted in 128 boxes handled by each subject $(8,320$ boxes in total). To be as close as possible to workplace conditions, no lifting technique or comment about the technique used was given to the participant.

As in a workplace, boxes had various characteristics: one $15-\mathrm{kg}$ box, one $23-\mathrm{kg}$ box, one weakened $15-$ $\mathrm{kg}$ box and one off-center 15-kg box (center of gravity $27 \mathrm{~cm}$ laterally from one side and $8 \mathrm{~cm}$ from the other), all of the same dimensions $(26 \mathrm{~cm}$ deep $\times 35 \mathrm{~cm}$ wide $\times 32 \mathrm{~cm}$ high). The weakened box contained 12 bottles of sand and water and had no cover so as to be deformable. To avoid constraining the grip, no instrumented handle was used. The evaluation of the method on standardized tasks with an instrumented handle was previously done (Muller et al., 2019a).

\subsection{Estimation of biomechanical variables}

The external forces prediction method is detailed in supplementary materials (Appendix A). For clarity, only the principal steps are summarized below.

All data from the prediction method were processed with CusToM (Customizable Toolbox for Musculoskeletal simulation (Muller et al., 2019b)). CusToM is a toolbox developed in Matlab ${ }^{\circledR}$ enabling musculoskeletal analyses based on inverse dynamics approaches with a high level of customization of the analysis.

A biomechanical model, composed of 16 rigid segments linked by 15 joints corresponding to 37 degrees of freedom, was used. The geometrical parameters were subject-specific calibrated using motion capture data and an optimization-based method (Muller et al. 2016). Body segment inertial parameters (BSIP) were extracted from anthropometric tables (Dumas et al., 2007). From the positions of the 48 anatomical landmarks (estimated from the positions of the 12 rigid clusters), the joint coordinates were computed in an inverse kinematics step (Lu and OConnor, 1999) and then filtered with a 4th-order Butterworth low-pass filter with a cutoff frequency of $5 \mathrm{~Hz}$ and no phase shift.

During the tasks, the external forces could be applied on the feet (GRF\&M) and hands (LCF\&M). Thus, a set of discrete contact points was defined on the biomechanical model: $N_{f}$ points under each foot and $N_{h}$ points 

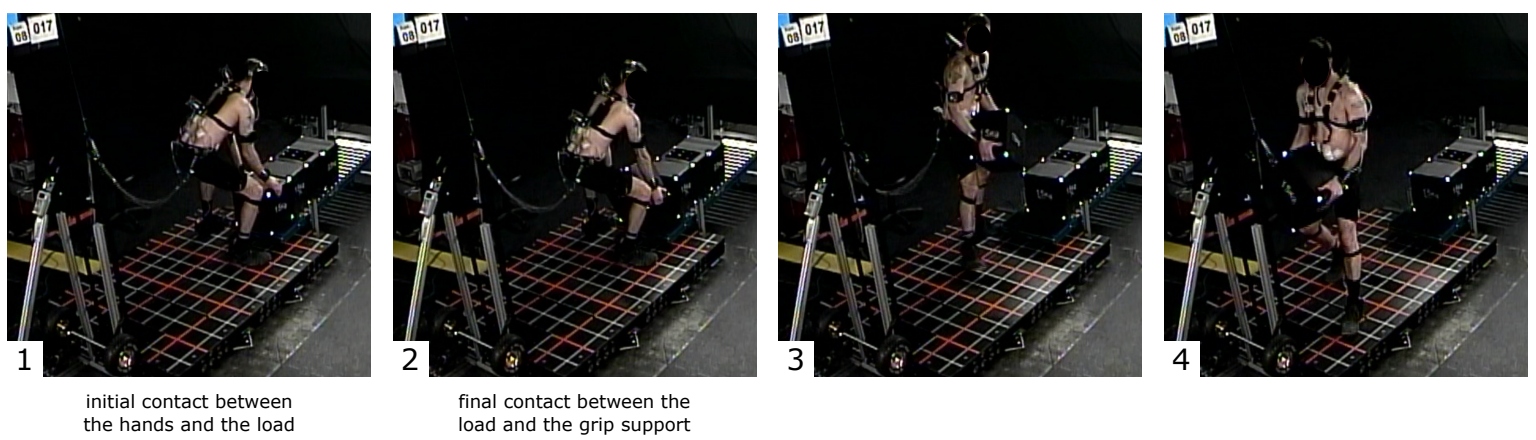
the hands and the load final contact between the final contact between the
load and the grip support
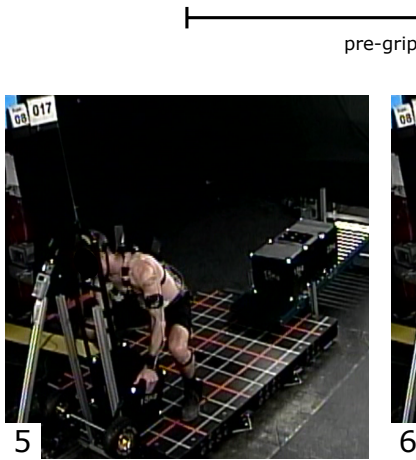

(1)

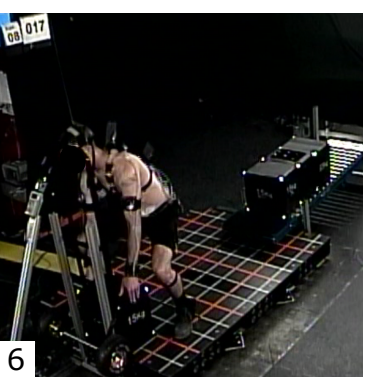

initial contact between the load and the destination support

final contact between the hands and the load

- - - - - L 1 post-deposit
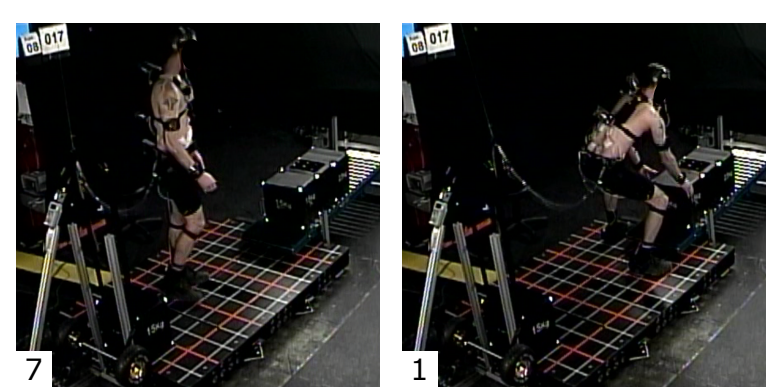

initial contact between the hands and the load

return

Figure 1: Different phases of the experimental task consisting in transferring four boxes one by one from a conveyor to a hand trolley and back again.

on each hand. For this study $N_{f}=14$ and $N_{h}=11$ were chosen. These points were located in order to map the contact area as proposed in previous studies (Fluit et al., 2014, Skals et al. 2017).

The handling task was composed of four different phases (Figure 1): 1) the pre-grip, from the initial contact between the hands and the load to the final contact between the load and the grip support; 2) the transfer, from the end of the pre-grip to the initial contact between the load and the destination support; 3) the postdeposit, from the end of the transfer to the final contact between the hands and the load; 4) the return, from the end of the post-deposit to the beginning of the pre-grip phase. During the pre-grip and post-deposit phases, the load was in contact with the subject's hands and load support. As no information was available about the contact between the load and its support, it was not possible to predict the external forces in these phases; consequently, they were not taken into account in this study, as discussed in the last section of the paper. Detection of the phases was accomplished manually using video recording.

During the return phase, as only external forces and moments were applied on the feet, the proposed method was a classical GRF\&M prediction method. Only the contact points defined under the feet were considered. At each instant, prediction of the external forces and moments was performed through an optimization procedure, which consisted in minimizing the sum of squared contact forces respecting the dynamics equations applied on the subject and additional constraints including Coulomb's law of friction and a maximal force for each contact point.

As the load grasp differed according the subject's technique or the experimental conditions, the orientation of the load in relation to the subject was not known. Thus, the inertial parameters of the load were not taken into account and only its mass was considered by estimating the location of the center of mass in the middle of the subject's wrist. Then, an inverse dynamics step was performed successively by using the predicted forces and moments and the measured values to obtain L5/S1 joint moments. The predicted and measured GRF\&M were used in a recursive Newton-Euler algorithm (Featherstone, 2014) with a bottom-up approach.

\subsection{Data analysis}

The pre-grip and post-deposit phases were excluded from the analysis and only the transfer and the return phases were considered. Four transfers with the box and three returns without the box were analyzed, totalling 2,080 tasks. For these phases, the method was evaluated by directly comparing the predicted and measured GRF\&M and also by comparing the L5/S1 joint 
moments computed with the predicted and measured GRF\&M.

Predicted and measured GRF\&M were compared with the Pearson correlation coefficient, computed for each transfer and return of each subject. Root mean squared error (RMSE) and relative RMSE (rRMSE) (Ren et al.,2008) were computed between the predicted and measured data. In the same manner, L5/S1 joint moments were compared with the Pearson correlation coefficient, RMSE and rRMSE. For the predicted $\left(u_{1}\right)$ and the measured data $\left(u_{2}\right)$ (instant $t$ varying from 0 to $T)$, RMSE and rRMSE were computed with Eq. (1) and Eq. (2), respectively.

$$
\begin{gathered}
R M S E=\frac{1}{T} \sum_{t=0}^{T} \sqrt{\left(u_{1}(t)-u_{2}(t)\right)^{2}} \\
r R M S E=\frac{R M S E}{\frac{1}{2} \sum_{i=1}^{2}\left(\max _{t \in[0, T]}\left(u_{i}(t)\right)-\min _{t \in[0, T]}\left(u_{i}(t)\right)\right)}
\end{gathered}
$$

For all the analyses, the antero-posterior axis was considered as the axis between the grip and the destination support. GRM were computed at the central point of the force platform.

\section{Results and discussion}

\subsection{GRFE $M$ estimation}

The Pearson correlation coefficient between predicted and measured GRF\&M are detailed in Table 1. A direct comparison of the results with those reported in the literature was not possible since different tasks were performed, only the orders of magnitude were compared. The minimal correlations were found along the vertical axis where the level of effort was higher. In comparison to the methods based on a contact model, except for the vertical GRF, correlations were higher. In comparison to the methods using machine learning, overall, the proposed method yielded lower correlations for the GRF (particularly on the vertical axis).

Table 2 and Table 3 show the RMSE and rRMSE between the predicted and measured GRF\&M. Also, the mean RMSE was $39 \mathrm{~N}( \pm 11), 17 \mathrm{~N}( \pm 6.5)$ and $14 \mathrm{~N}$ $( \pm 5.2)$ respectively for the vertical, antero-posterior and medio-lateral GRF and $24 \mathrm{Nm}( \pm 10), 17 \mathrm{Nm}( \pm 6.7)$ and $7.7 \mathrm{Nm}( \pm 2.4)$ respectively for the sagittal, frontal and transverse GRM. Considering the vertical axis (concerned with the highest force magnitudes), even if contact models were added on each hand, a comparison of results shows the same magnitude as in the literature with the same type of method. Higher errors are only observed in Skals et al. (2017) where large dynamic motions were studied. Considering the other directions, the correlations were higher than or comparable to those reported in the literature. However, the variety of motions studied in the different papers makes it difficult to directly compare results. Contrary to other tasks, MMH tasks have no preferred direction within a large movement area. For example in the case of GRF, the correlation was higher, whereas the RMSE was the same magnitude or higher than reported in the literature. The mean RMSE for GRM was higher overall than the literature results; however, the corresponding rRMSE was lower since the amplitude of the GRM was more important in the current study. The large amplitude is explained by the large displacements made during the MMH tasks.

\subsection{L5/S1 joint moment estimation}

The results of comparison of L5/S1 joint moments with predicted and measured GRF\&M is detailed in Table 4 Higher correlation was observed on the sagittal axis (corresponding to the flexion/extension) where the moment values were the most important. By comparing a bottom-up and a top-down approach during lifting tasks, Larivière and Gagnon (1998) reported uncertainties of $15 \mathrm{Nm}, 8 \mathrm{Nm}$ and $8 \mathrm{Nm}$ for the L5/S1 joint moment estimation along the same respective axis. In a similar study, Plamondon et al. (1996) observed uncertainties of $4 \mathrm{Nm}, 4 \mathrm{Nm}$ and $5 \mathrm{Nm}$ along the same axis. Kingma et al. (1996) reported uncertainties in the range of $3.9 \mathrm{Nm}$ to $23.7 \mathrm{Nm}$ on the sagittal axis, $5.4 \mathrm{Nm}$ to $20.5 \mathrm{Nm}$ on the frontal axis and $1.9 \mathrm{Nm}$ to $15.2 \mathrm{Nm}$ on the transverse axis. Given the tasks studied and models used, the uncertainties in the literature varied greatly. However, the errors found were comparable to the estimation uncertainties reported by Larivière and Gagnon (1998) and were in the range of uncertainties reported by Kingma et al. (1996).

Considering the transfer and return phases separately, the RMSE were $14.0 \mathrm{Nm}( \pm 6.9)$ and $14.1 \mathrm{Nm}$ $( \pm 6.5)$ for the sagittal axis, $10.0 \mathrm{Nm}( \pm 4.0)$ and $9.0 \mathrm{Nm}$ $( \pm 4.0)$ for the frontal axis and $10.0 \mathrm{Nm}( \pm 4.0)$ and $8.0 \mathrm{Nm}$ $( \pm 3.5)$ for the transverse axis. Thus, compared to a classical GRF\&M prediction method with no box, taking into account a load does not add any error in the estimation of L5/S1 joint moments.

Generally, phases where prediction had poorer results were observed when the proposed contact model was the least representative of the performed movement. Two recurrent observations were noted. First, several subjects threw the load before contact with the destination support, whereas the model assumes that the load is always between the subject's hands. Second, in several cases, there was contact between the load and a body part other than the hands (in most cases the belly). For these situations, the model differs considerably from the experimental conditions and the predicted data reveal more significant errors. 
Table 1: Pearson correlation coefficient $r( \pm \mathrm{SD})$ between predicted and measured GRF\&M along each direction computed and averaged for each transfer and return phases. Results of the proposed method are compared to those detailed in the literature: three methods based on a contact model (Fluit et al. 2014. Skals et al. 2017| Larsen and Svenningsen 2018), and two methods based on machine learning (Oh et al. 2013 Johnson et al. 2018). For the studies of Fluit et al. (2014), Skals et al. (2017) and Larsen and Svenningsen (2018), the results for the different types of motions were averaged. V: vertical; AP: antero-posterior; ML: medio-lateral; S: sagittal; F: frontal; T: transverse.

\begin{tabular}{|c|c|c|c|c|c|c|c|}
\hline & \multirow{2}{*}{ Motions } & \multicolumn{3}{|c|}{ GRF } & \multicolumn{3}{|c|}{ GRM } \\
\hline & & $\mathrm{V}$ & AP & ML & $\mathrm{S}$ & $\mathrm{F}$ & $\mathrm{T}$ \\
\hline Proposed method & MMH & $\begin{array}{c}0.91 \\
(0.08)\end{array}$ & $\begin{array}{c}0.95 \\
(0.03)\end{array}$ & $\begin{array}{c}0.94 \\
(0.08)\end{array}$ & $\begin{array}{c}0.99 \\
(0.01)\end{array}$ & $\begin{array}{c}0.98 \\
(0.05)\end{array}$ & $\begin{array}{c}0.91 \\
(0.07)\end{array}$ \\
\hline Fluit et al. (2014) & Gait & 0.96 & 0.95 & 0.81 & 0.84 & 0.65 & 0.54 \\
\hline Fluit et al. (2014) & $\begin{array}{l}\text { Squat and } \\
\text { stairs }\end{array}$ & 0.82 & 0.42 & 0.44 & 0.66 & 0.58 & -0.02 \\
\hline Skals et al. (2017) & Sports & 0.98 & 0.85 & 0.64 & 0.85 & 0.67 & 0.28 \\
\hline$\frac{\text { Larsen and Svenningsen }}{(2018)}$ & MMH & 0.94 & 0.53 & 0.71 & 0.64 & 0.76 & 0.24 \\
\hline Oh et al. (2013) & Gait & 0.99 & 0.98 & 0.92 & 0.99 & 0.84 & 0.87 \\
\hline Johnson et al. (2018) & Sidestep & 0.99 & 0.97 & 0.98 & 0.94 & 0.88 & 0.92 \\
\hline
\end{tabular}

\subsection{General discussion}

On the basis of these results, the proposed method is very encouraging. It allows MMH tasks to be studied without the use of force platforms, which increases the ecological aspect of the tasks. In this paper, the predicted external forces and moments were used to estimate the L5/S1 joint moments. However, the method can also be used to compute several biomechanical variables permitting assessment of handling tasks, for example destabilizing and stabilizing forces to assess equilibrium (Duclos et al., 2009) or glenohumeral joint moments to assess shoulder loading (which cannot be estimated using only force platforms on asymmetrical tasks). Compared to previous studies, the current method was evaluated under experimental conditions representative of the workplace: variability of the subjects (men / women, healthy bodyweight / obese, experts / novices), transporting boxes from a conveyor to a hand trolley, no constraint on foot motion, and no particular instructions given. Even if machine learning methods have overall better results, they require a learning phase using a data set corresponding to external forces and moments on the standardized motion, which is not the situation studied here. In the context of MMH tasks, machine learning seems difficult to adapt since the tasks vary widely, i.e. variability of movement, handling techniques and carried loads. The learning phase would require a very large data set (Halilaj et al. 2018).

The results obtained appear satisfactory despite several hypotheses where no specific parameters were chosen for the different models. First, the load model was simplified since its inertial parameters and the specific location of the center of mass of the off-center box and the weakened box were not taken into account. Using only the mass of the load therefore simplifies the method development in an ecological context by us- ing various loads. For example, for order picking in a bonded warehouse, the mass of the load could easily be obtained from the order tracking data. Second, the BSIP were based on a regression method (Dumas et al. 2007) even though some of the cohort were obese workers (greater than $50^{\text {th }}$ percentile). Moreover, using only regression methods allows a simplification of the experimental protocol where only the subject mass is used, which facilitates use of the method in an applied ergonomics context.

\subsection{Limits and perspectives}

The current method has several limitations. First, it was based on an optoelectronical motion capture system which is usually only available in motion analysis labs. Where the aim is to improve the ecological aspect of the experiments, wearable motion capture systems could be used. For example, inertial motion capture systems (Vignais et al. 2013, Robert-Lachaine et al., 2017; Faber et al. 2018) or depth cameras (Plantard et al. 2017a b) have already been proposed for workstation ergonomic posture assessments. More recently, in the laboratory, the use of 2D images associated to deep learning methods were also developed to estimate pose during lifting tasks (Mehrizi et al., 2019). The extension of the proposed method to include this type of technology is one important means to complete an ergonomics assessment in the workplace. Second, the phases of the MMH tasks were identified manually by using video recordings, which requires a timeconsuming post-treatment. The addition of sensors (for example on the load or on the hands) or the use of an automatic identification method could decrease the post-treatment time. Third, acquisition frequency has a direct impact on the computation of dynamic quantities and therefore on prediction forces. If very dynamic 
Table 2: Averaged RMSE $( \pm \mathrm{SD})$ and rRMSE $( \pm \mathrm{SD})$ between predicted and measured GRF along each direction. Results of the proposed method are compared to those detailed in the literature: four methods based on a contact model (Fluit et al. 2014. Skals et al. 2017, Larsen and Svenningsen 2018. Muller et al. 2019a) and one method based on machine learning (Oh et al. 2013). For the studies of Fluit et al. (2014), Skals et al. (2017) and Larsen and Svenningsen (2018), the results for the different types of motions were averaged. The results are presented first normalized by body weight and second divided by the subject's mass, which improves comparison to the literature where results are available. - refers to unavailable data. V: vertical; AP: antero-posterior; ML: medio-lateral.

\begin{tabular}{|c|c|c|c|c|c|c|c|}
\hline \multirow[b]{3}{*}{ Proposed method } & \multirow[b]{2}{*}{ Motions } & \multicolumn{2}{|c|}{$\mathrm{V}$} & \multicolumn{2}{|c|}{ AP } & \multicolumn{2}{|c|}{$\mathrm{ML}$} \\
\hline & & $\begin{array}{l}\text { RMSE } \\
(\mathrm{N} / \mathrm{kg})\end{array}$ & $\begin{array}{l}\text { rRMSE } \\
(\%)\end{array}$ & $\begin{array}{l}\text { RMSE } \\
(\mathrm{N} / \mathrm{kg})\end{array}$ & $\begin{array}{l}\text { rRMSE } \\
\qquad(\%)\end{array}$ & $\begin{array}{l}\text { RMSE } \\
(\mathrm{N} / \mathrm{kg})\end{array}$ & $\begin{array}{l}\text { rRMSE } \\
\quad(\%)\end{array}$ \\
\hline & ММH & $\begin{array}{c}0.51 \\
(0.14)\end{array}$ & $\begin{array}{c}7.1 \\
(2.2)\end{array}$ & $\begin{array}{c}0.22 \\
(0.08)\end{array}$ & $\begin{array}{c}4.7 \\
(1.3)\end{array}$ & $\begin{array}{c}0.19 \\
(0.06)\end{array}$ & $\begin{array}{c}5.3 \\
(1.9)\end{array}$ \\
\hline Fluit et al. (2014) & Gait & 0.68 & - & 0.30 & - & 0.19 & - \\
\hline Fluit et al. (2014) & $\begin{array}{l}\text { Squat and } \\
\text { stairs }\end{array}$ & 0.70 & - & 0.24 & - & 0.29 & - \\
\hline Skals et al. (2017) & Sport & 1.05 & - & 0.61 & - & 0.39 & - \\
\hline $\begin{array}{l}\text { Larsen and Svenningsen } \\
\qquad(2018)\end{array}$ & $\mathrm{MMH}$ & 0.60 & 10.4 & 0.46 & 58.2 & 0.13 & 21.4 \\
\hline Muller et al. (2019a) & $\mathrm{MMH}$ & 0.24 & 2.86 & 0.08 & 16.7 & 0.40 & 37.8 \\
\hline Oh et al. (2013) & Gait & $\begin{array}{c}0.65 \\
(0.18)\end{array}$ & $\begin{array}{c}5.8 \\
(1.0)\end{array}$ & $\begin{array}{c}0.15 \\
(0.06)\end{array}$ & $\begin{array}{c}7.3 \\
(0.8)\end{array}$ & $\begin{array}{c}0.04 \\
(0.02)\end{array}$ & $\begin{array}{l}10.9 \\
(1.8)\end{array}$ \\
\hline
\end{tabular}

phases (pre-grip, grip, deposit or post-deposit phases) are of interest, an acquisition frequency higher than $30 \mathrm{~Hz}$ may be necessary. In addition, in these phases, a more detailed modelling of the hypothesized contact points would improve prediction. Fourth, a more detailed modelling of the discrete contact points would improve prediction method. In the current study, the contacts points were chosen to map uniformly the hand surface, with a similar density to the ones on the feet. A sensitivity study to determine the optimal number of points to use and their location on the hand would improve the model. Finally, since the method is based on a contact model, the pre-grip and post-deposit phases were not taken into account. Given the tasks studied, assessing these phases could be of interest. In this case, the addition of sensors on the ground or on the load appears necessary.

\section{Conclusion}

The proposed method showed that external forces and moments and back loading can be estimated during manual material handling tasks from only motion data on a wide variety of subjects (experts / novice, men / women, healthy bodyweight / obese). This method improves the ecological aspect of the tasks by not constraining the movement area or the handling techniques. Handler follow-up could thus be improved by assessing the ergonomics of MMH tasks that are as representative as possible of those performed in the workplace.

\section{References}

Choi, A., Lee, J.-M., Mun, J. H., 2013. Ground reaction forces predicted by using artificial neural network during asymmetric movements. International Journal of Precision Engineering and Manufacturing 14 (3), 475-483.

Corbeil, P., Plamondon, A., Handrigan, G., Vallée-Marcotte, J., Laurendeau, S., Ten Have, J., Manzerolle, N., 2019. Biomechanical analysis of manual material handling movement in healthy weight and obese workers. Applied ergonomics 74, 124-133.

Corbeil, P., Plamondon, A., Teasdale, N., Handrigan, G., 2013. Biomechanical differences between obese and healthy-weight workers in manual materials handling. In: Proceedings of the human factors and ergonomics society annual meeting. Vol. 57. SAGE Publications Sage CA: Los Angeles, CA, pp. 958-961.

Delisle, A., Gagnon, M., Desjardins, P., 1999. Kinematic analysis of footstep strategies in asymmetrical lifting and lowering tasks. International Journal of Industrial Ergonomics 23 (5-6), 451-460.

Desjardins, P., Gagnon, M., 2001. A force platform for large human displacements. Medical engineering \& physics 23 (2), 143-146.

Dijkstra, E. J., Gutierrez-Farewik, E. M., 2015. Computation of ground reaction force using zero moment point. Journal of biomechanics 48 (14), 3776-3781.

Duclos, C., Desjardins, P., Nadeau, S., Delisle, A., Gravel, D., Brouwer, B., Corriveau, H., 2009. Destabilizing and stabilizing forces to assess equilibrium during everyday activities. Journal of biomechanics 42 (3), 379-382.

Dumas, R., Cheze, L., Verriest, J.-P., 2007. Adjustments to mcconville et al. and young et al. body segment inertial parameters. Journal of biomechanics 40 (3), 543-553.

Faber, G., Chang, C., Kingma, I., Dennerlein, J., van Dieën, J., 2016. Estimating 3D L5/S1 moments and ground reaction forces during trunk bending using a full-body ambulatory inertial motion capture system. Journal of biomechanics 49 (6), 904-912.

Faber, G. S., Koopman, A., Kingma, I., Chang, C., Dennerlein, J., van Dieën, J., 2018. Continuous ambulatory hand force monitoring during manual materials handling using instrumented force shoes and an inertial motion capture suit. Journal of biomechanics 70, 235-241.

Featherstone, R., 2014. Rigid body dynamics algorithms. Springer. Fluit, R., Andersen, M. S., Kolk, S., Verdonschot, N., Koopman, H. F., 2014. Prediction of ground reaction forces and moments 
Table 3: Averaged RMSE $( \pm$ SD) and rRMSE $( \pm$ SD) between predicted and measured GRM along each direction. The proposed method results are compared to those detailed in the literature: three methods based on a contact model (Fluit et al. 2014, Larsen and Svenningsen. 2018 Muller et al. 2019a) and one method based on machine learning (Oh et al. 2013). For the studies of Fluit et al. (2014) and Larsen and Svenningsen (2018), the results for the different types of motion were averaged. The results are presented first normalized by body weight times height and second divided by the subject's mass, which improves the comparison to the literature according to the available results. refers to unavailable data. S: sagittal; F: frontal; T: transverse.

\begin{tabular}{|c|c|c|c|c|c|c|c|}
\hline \multirow[b]{3}{*}{ Proposed method } & Motions & \multicolumn{2}{|c|}{$\mathrm{S}$} & \multicolumn{2}{|c|}{$\mathrm{F}$} & \multicolumn{2}{|c|}{$\mathrm{T}$} \\
\hline & IVIOtions & $\begin{array}{l}\text { RMSE } \\
\mathrm{Nm} / \mathrm{kg}\end{array}$ & $\begin{array}{l}\text { rRMSE } \\
\%\end{array}$ & $\begin{array}{l}\text { RMSE } \\
\mathrm{Nm} / \mathrm{kg}\end{array}$ & $\begin{array}{l}\mathrm{rRMSE} \\
\%\end{array}$ & $\begin{array}{l}\text { RMSE } \\
\mathrm{Nm} / \mathrm{kg}\end{array}$ & $\begin{array}{c}\text { rRMSE } \\
\%\end{array}$ \\
\hline & $\mathrm{MMH}$ & $\begin{array}{c}0.32 \\
(0.12)\end{array}$ & $\begin{array}{c}2.0 \\
(0.80)\end{array}$ & $\begin{array}{c}0.22 \\
(0.07)\end{array}$ & $\begin{array}{c}3.4 \\
(1.7)\end{array}$ & $\begin{array}{c}0.10 \\
(0.03)\end{array}$ & $\begin{array}{c}7.2 \\
(2.4)\end{array}$ \\
\hline Fluit et al. (2014) & Gait & 0.19 & - & 0.09 & - & 0.20 & - \\
\hline Fluit et al. 2014) & $\begin{array}{l}\text { Squat and } \\
\text { stairs }\end{array}$ & 0.17 & - & 0.14 & - & 0.14 & - \\
\hline $\begin{array}{c}\text { Larsen and Svenningsen } \\
(2018)\end{array}$ & MMH & 0.08 & 28.5 & 0.12 & 19.8 & 0.10 & 64.1 \\
\hline Muller et al. (2019a) & $\mathrm{MMH}$ & 0.14 & 13.2 & 0.09 & 16.8 & 0.09 & 57.1 \\
\hline Oh et al. (2013) & Gait & $\begin{array}{c}0.08 \\
(0.04)\end{array}$ & $\begin{array}{c}9.9 \\
(1.9)\end{array}$ & $\begin{array}{c}0.05 \\
(0.03)\end{array}$ & $\begin{array}{c}22.8 \\
(22.8)\end{array}$ & $\begin{array}{c}0.03 \\
(0.02)\end{array}$ & $\begin{array}{l}25.5 \\
(4.5)\end{array}$ \\
\hline
\end{tabular}

Table 4: Comparison of L5/S1 joint moments along each direction computed with predicted and with measured GRF\&M using the Pearson correlation coefficient $r( \pm \mathrm{SD})$ and the RMSE $( \pm \mathrm{SD})$, where $r$ was computed and averaged for each transfer and return phases. S: sagittal; F: frontal; T: transverse.

\begin{tabular}{cccc}
\hline & $\mathrm{S}$ & $\mathrm{F}$ & $\mathrm{T}$ \\
\cline { 2 - 4 }$r$ & 0.947 & 0.875 & 0.794 \\
& $(0.072)$ & $(0.111)$ & $(0.136)$ \\
RMSE (Nm) & 14.1 & 9.6 & 9.1 \\
& $(6.7)$ & $(4.3)$ & $(4.1)$ \\
rRMSE $(\%)$ & 5.3 & 5.9 & 8.0 \\
& $(2.5)$ & $(2.7)$ & $(3.3)$ \\
\hline
\end{tabular}

during various activities of daily living. Journal of biomechanics 47 (10), 2321-2329.

Gagnon, D., Plamondon, A., Larivière, C., 2018. A comparison of lumbar spine and muscle loading between male and female workers during box transfers. Journal of biomechanics 81, 76-85.

Halilaj, E., Rajagopal, A., Fiterau, M., Hicks, J. L., Hastie, T. J., Delp, S. L., 2018. Machine learning in human movement biomechanics: best practices, common pitfalls, and new opportunities. Journal of biomechanics.

Johnson, W. R., Mian, A., Donnelly, C. J., Lloyd, D., Alderson, J., 2018. Predicting athlete ground reaction forces and moments from motion capture. Medical \& biological engineering \& computing 56 (10), 1781-1792.

Jung, Y., Jung, M., Ryu, J., Yoon, S., Park, S.-K., Koo, S., 2016. Dynamically adjustable foot-ground contact model to estimate ground reaction force during walking and running. Gait \& posture $45,62-68$.

Kingma, I., de Looze, M. P., Toussaint, H. M., Klijnsma, H. G., Bruijnen, T. B., 1996. Validation of a full body 3-d dynamic linked segment model. Human Movement Science 15 (6), 833-860.

Koopman, B., Grootenboer, H. J., De Jongh, H. J., 1995. An inverse dynamics model for the analysis, reconstruction and prediction of bipedal walking. Journal of biomechanics 28 (11), 1369-1376.

Larivière, C., Gagnon, D., 1998. Comparison between two dynamic methods to estimate triaxial net reaction moments at the L5/S1 joint during lifting. Clinical Biomechanics 13 (1), 36-47.
Larsen, F. G., Svenningsen, F. P., 2018. Estimation of spinal loading using inertial motion capture and ground reaction force prediction: A validation study. Master's thesis, Faculty of Health Science and Technology, Aalborg University.

Lu, T.-W., OConnor, J., 1999. Bone position estimation from skin marker co-ordinates using global optimisation with joint constraints. Journal of biomechanics 32 (2), 129-134.

Marras, W., Parakkat, J., Chany, A., Yang, G., Burr, D., Lavender, S., 2006. Spine loading as a function of lift frequency, exposure duration, and work experience. Clinical Biomechanics 21 (4), 345352.

Mehrizi, R., Peng, X., Xu, X., Zhang, S., Li, K., 2019. A deep neural network-based method for estimation of 3D lifting motions. Journal of biomechanics 84, 87-93.

Muller, A., Germain, C., Pontonnier, C., Dumont, G., 2016. A simple method to calibrate kinematical invariants: application to overhead throwing. In: ISBS-Conference Proceedings Archive. Vol. 33.

Muller, A., Pontonnier, C., Dumont, G., 2019a. Motion-based prediction of hands and feet contact efforts during asymmetric handling tasks. IEEE Transactions on Biomedical Engineering, 1-11.

Muller, A., Pontonnier, C., Puchaud, P., Dumont, G., 2019b. CusToM: a Matlab toolbox for musculoskeletal simulation. Journal of Open Source Software 4 (33), 1-3.

National Research Council, 2001. Musculoskeletal disorders and the workplace: low back and upper extremities. The National Academies Press, Washington, DC

Ning, X., Zhou, J., Dai, B., Jaridi, M., 2014. The assessment of material handling strategies in dealing with sudden loading: The effects of load handling position on trunk biomechanics. Applied ergonomics 45 (6), 1399-1405.

Oh, S. E., Choi, A., Mun, J. H., 2013. Prediction of ground reaction forces during gait based on kinematics and a neural network model. Journal of biomechanics 46 (14), 2372-2380.

Plamondon, A., Denis, D., Delisle, A., Larivière, C., Salazar, E., research group, I. M., et al., 2010. Biomechanical differences between expert and novice workers in a manual material handling task. Ergonomics 53 (10), 1239-1253.

Plamondon, A., Gagnon, M., Desjardins, P., 1996. Validation of two 3 -D segment models to calculate the net reaction forces and moments at the L5S1 joint in lifting. Clinical Biomechanics 11 (2), 101-110.

Plamondon, A., Larivière, C., Delisle, A., Denis, D., Gagnon, D., 
2012. Relative importance of expertise, lifting height and weight lifted on posture and lumbar external loading during a transfer task in manual material handling. Ergonomics 55 (1), 87-102.

Plantard, P., Muller, A., Pontonnier, C., Dumont, G., Shum, H. P., Multon, F., 2017a. Inverse dynamics based on occlusion-resistant kinect data: Is it usable for ergonomics? International Journal of Industrial Ergonomics 61, 71-80.

Plantard, P., Shum, H. P., Le Pierres, A.-S., Multon, F., 2017b. Validation of an ergonomic assessment method using kinect data in real workplace conditions. Applied ergonomics 65, 562-569.

Ren, L., Jones, R. K., Howard, D., 2008. Whole body inverse dynamics over a complete gait cycle based only on measured kinematics. Journal of biomechanics 41 (12), 2750-2759.

Robert-Lachaine, X., Mecheri, H., Larue, C., Plamondon, A., 2017. Validation of inertial measurement units with an optoelectronic system for whole-body motion analysis. Medical \& biological engineering \& computing 55 (4), 609-619.

Skals, S., Jung, M. K., Damsgaard, M., Andersen, M. S., 2017. Prediction of ground reaction forces and moments during sportsrelated movements. Multibody system dynamics 39 (3), 175-195.

Vignais, N., Miezal, M., Bleser, G., Mura, K., Gorecky, D., Marin, F., 2013. Innovative system for real-time ergonomic feedback in industrial manufacturing. Applied ergonomics 44 (4), 566-574. 